\title{
Tellurite-mediated disabling of [4Fe-4S] clusters of Escherichia coli dehydratases
}

\author{
Correspondence \\ Claudio C. Vásquez \\ claudio.vasquez@usach.cl
}

Received 28 November 2008

Revised 27 February 2009

Accepted 9 March 2009

\author{
Iván L. Calderón,† Alex O. Elías, Eugenia L. Fuentes, Gonzalo A. Pradenas, \\ Miguel E. Castro, Felipe A. Arenas, José M. Pérez and Claudio C. Vásquez
}

Laboratorio de Microbiología Molecular, Departamento de Biología, Facultad de Química y Biología, Universidad de Santiago de Chile, Santiago, Chile

\begin{abstract}
The tellurium oxyanion tellurite is toxic for most organisms and it seems to alter a number of intracellular targets. In this work the toxic effects of tellurite upon Escherichia coli [4Fe-4S] cluster-containing dehydratases was studied. Reactive oxygen species (ROS)-sensitive fumarase $A(F u m A)$ and aconitase $B(A c n B)$ as well as ROS-resistant fumarase C (FumC) and aconitase A (AcnA) were assayed in cell-free extracts from tellurite-exposed cells in both the presence and absence of oxygen. While over $90 \%$ of FumA and AcnB activities were lost in the presence of oxygen, no enzyme inactivation was observed in anaerobiosis. This result was not dependent upon protein biosynthesis, as determined using translation-arrested cells. Enzyme activity of purified FumA and AcnB was inhibited when exposed to an in vitro superoxide-generating, telluritereducing system (ITRS). No inhibitory effect was observed when tellurite was omitted from the ITRS. In vivo and in vitro reconstitution experiments with tellurite-damaged FumA and AcnB suggested that tellurite effects involve [Fe-S] cluster disabling. In fact, after exposing FumA to ITRS, released ferrous ion from the enzyme was demonstrated by spectroscopic analysis using the specific $\mathrm{Fe}^{2+}$ chelator 2,2'-bipyridyl. Subsequent spectroscopic paramagnetic resonance analysis of FumA exposed to ITRS showed the characteristic signal of an oxidatively inactivated $[3 \mathrm{Fe}-4 \mathrm{~S}]^{+}$cluster. These results suggest that tellurite inactivates enzymes of this kind via a superoxide-dependent disabling of their $[4 \mathrm{Fe}-4 \mathrm{~S}]$ catalytic clusters.
\end{abstract}

\section{INTRODUCTION}

Since no biological role of tellurium is known to date, scientific interest on it has focused mainly on its toxicity. Tellurite $\left(\mathrm{TeO}_{3}^{2-}\right)$, one of the most oxidized forms of tellurium, is toxic to both prokaryotes and eukaryotes. Gram-negative bacteria are particularly sensitive to tellurium salts, whereas some Gram-positive species exhibit natural resistance to tellurite (Taylor, 1999). Experimental evidence accumulated during the last few years suggests that tellurite exerts its toxicity, at least in part, through the generation of reactive oxygen species (ROS) (Borsetti et al., 2005; Calderón et al., 2006; Pérez et al., 2007; Tremaroli et al., 2006).

Tellurite-mediated ROS generation was first suggested by studies with tellurite-hypersensitive Escherichia coli $\operatorname{sod} A \operatorname{sod} B$ strains and by the observation that minimal

†Present address: Facultad de Ciencias de la Salud, Universidad Andrés Bello, Santiago, Chile.

Abbreviations: ITRS, in vitro tellurite-reducing system; MV, methyl viologen; ROS, reactive oxygen species; SOD, superoxide dismutase; TBAR, thiobarbituric acid-reactive substance.

Four supplementary figures are available with the online version of this paper. inhibitory concentrations of tellurite were higher for cells grown under anaerobic conditions (Tantaleán et al., 2003). The leading ROS generated as consequence of tellurite reduction was shown to be superoxide $\left(\mathrm{O}_{2}^{-}\right)$(Calderón et al., 2006). Recent work from our laboratory showed that tellurite-exposed E. coli exhibits increased cytoplasmic ROS, protein carbonyl content, thiobarbituric acid-reactive substances (TBARs), and superoxide dismutase (SOD) and catalase activities (Pérez et al., 2007).

Various dehydratases involved in oxidative metabolism possess catalytic [ $4 \mathrm{Fe}-4 \mathrm{~S}]$ clusters at their active sites that facilitate the binding and consequent dehydration of their substrates (Storz \& Imlay, 1999). Solvent exposure of these clusters renders them vulnerable to superoxide, which is electrostatically attracted to the catalytic $\mathrm{Fe}$ atom of the cluster. Examples of such enzymes - which are commonly used as markers of oxidative damage - include fumarases A (FumA) and B (FumB) (Liochev \& Fridovich, 1993), aconitase B (AcnB) (Gardner \& Fridovich, 1991) and dihydroxyacid dehydratase (Storz \& Imlay, 1999; Kuo et al., 1987). Loss of $\mathrm{Fe}^{2+}$ by univalent oxidation of their $[4 \mathrm{Fe}-4 \mathrm{~S}]^{2+}$ centres results in the generation of a $[3 \mathrm{Fe}-$ $4 \mathrm{~S}]^{+}$cluster with a concomitant loss of enzyme activity (Imlay, 2003). 
In addition to FumA, FumB and AcnB E. coli encodes ROSresistant isoforms fumarase $\mathrm{C}$ (FumC) and aconitase A (AcnA). Unlike FumA and FumB, FumC has no iron-sulfur centre and is fully resistant to superoxide. Its induction mediated by the SoxRS regulon allows some flux through the tricarboxylic acid cycle when FumA and FumB have been damaged by superoxide (Liochev \& Fridovich, 1993). AcnA is also induced upon activation of soxRS under oxidative stress conditions, thus compensating for oxidative damage of the housekeeping AcnB (Varghese et al., 2003).

We have previously observed that tellurite exposure results in decreased AcnB and FumA activities in E. coli (Pérez et al., 2007). To shed further light on bacterial tellurite toxicity and because of the physiological relevance of this type of dehydratase, in this work we studied the effect of tellurite on $[4 \mathrm{Fe}-4 \mathrm{~S}]^{2+}$ cluster-containing enzymes using $E$. coli AcnB and FumA as models. Using genetic, chemical and biochemical approaches here we show that tellurite is particularly harmful for these dehydratases and that tellurite damage seems to be indirect. Enzyme inactivation appears to result from superoxide generation that occurs during intracellular tellurite reduction and involves $\mathrm{Fe}^{+2}$ release from the catalytic $[4 \mathrm{Fe}-4 \mathrm{~S}]^{2+}$ clusters.

\section{METHODS}

Bacterial strains and growth conditions. Bacterial strains used in this study are listed in Table 1. Bacteria were routinely grown in LB medium (Sambrook et al., 1989) at $37{ }^{\circ} \mathrm{C}$ with vigorous shaking. Gene induction was carried out in the presence of $0.2 \%$ arabinose. When needed ampicillin $\left(100 \mu \mathrm{g} \mathrm{ml}^{-1}\right)$, chloramphenicol $(50 \mu \mathrm{g}$ $\left.\mathrm{ml}^{-1}\right)$ or kanamycin $\left(100 \mu \mathrm{g} \mathrm{ml}^{-1}\right)$ was added to the medium. Growth under anaerobic conditions used a nitrogen-purged Difco anaerobic chamber.

Enzyme activity. In general, enzyme activity was expressed as percentage activity, $100 \%$ being the activity of the enzyme in extracts of cells not exposed to $\mathrm{K}_{2} \mathrm{TeO}_{3}$ at zero time. Total fumarase activity was determined by the formation of fumarate from L-malate at $250 \mathrm{~nm}$ for $2 \mathrm{~min}$ in a mix $(1 \mathrm{ml})$ that contained $50 \mathrm{mM}$ potassium phosphate buffer, $\mathrm{pH}$ 7.0, $50 \mu \mathrm{M}$ L-malate and the extract (15 $\mu \mathrm{g}$ protein). FumC activity was measured after preincubating crude extracts for $2 \mathrm{~h}$ at
$37{ }^{\circ} \mathrm{C}$ to inactivate endogenous FumA and FumB. A unit of FumC activity was defined as the amount of enzyme that converts $0.5 \mu \mathrm{mol} L-$ malate $\min ^{-1}$ into fumarate at $25^{\circ} \mathrm{C}\left(\varepsilon=1.62 \mathrm{mM}^{-1} \mathrm{~cm}^{-1}\right)$.

Aconitase activity was determined as the production of cis-aconitate from citrate at $240 \mathrm{~nm}$ as described by Varghese et al. (2003). A unit of aconitase activity was defined as the amount of enzyme that generates $0.5 \mu \mathrm{mol}$ cis-aconitate $\mathrm{min}^{-1}$ at $25^{\circ} \mathrm{C}\left(\varepsilon=3.6 \mathrm{mM}^{-1} \mathrm{~cm}^{-1}\right)$.

qRT-PCR analysis. Total RNA was purified using the RNAsy kit (Qiagen). Briefly, E. coli was inoculated in $200 \mathrm{ml} \mathrm{LB}$ medium, grown to $\mathrm{OD}_{600} \sim 0.6$ and cultures were continued for $15 \mathrm{~min}$ after amending with potassium tellurite $\left(0.5 \mu \mathrm{g} \mathrm{ml}^{-1}\right)$ or methyl viologen $\left(\mathrm{MV}, 100 \mu \mathrm{g} \mathrm{ml}^{-1}\right)$.

Two micrograms of purified RNA was used as template for qRT-PCR. Reactions were performed using the LightCycler RNA Amplification SYBR Green I kit (Roche Applied Science) as recommended by the vendor. Transcript amount (ng) for each gene was calculated from a standard curve made with known template concentrations. Specific primers used are listed in Table 2.

In vitro tellurite-reducing system (ITRS) and superoxide generation. The tellurite-reducing reaction mix consisted of $50 \mathrm{mM}$ Tris/HCl buffer, pH 7.4, containing $1 \mathrm{mM} \mathrm{K}_{2} \mathrm{TeO}_{3}, 1 \mathrm{mM}$ $\mathrm{NADH}$ and $0.7 \mathrm{mg}$ bovine liver catalase $\mathrm{ml}^{-1}$ (Sigma). Standard reactions were incubated at $37{ }^{\circ} \mathrm{C}$ for $30 \mathrm{~min}$ as described earlier (Calderón et al., 2006). A positive superoxide-generating control used $50 \mathrm{mM}$ Tris/ $\mathrm{HCl}$ buffer, $\mathrm{pH} 7.4$, containing $0.05 \mathrm{mM}$ xanthine (Sigma) and $1.6 \mathrm{mU}$ xanthine oxidase $\mathrm{ml}^{-1}$ (Sigma).

Cloning, overexpression and purification of FumA. The E. coli fumA gene was amplified by PCR (Table 2), purified, cloned in pETTOPO/101 (Invitrogen) and transformed into E. coli TOP10. Purified recombinant plasmid pET-fumA was introduced into E. coli BL21(DE3) by electroporation.

E. coli BL21(DE3) carrying pET-fumA was induced with $1 \mathrm{mM}$ IPTG for $5 \mathrm{~h}$ and FumA was purified by affinity chromatography on HisTrap HP (Amersham) columns according to the vendor's instructions. Protein concentration was determined by the method of Bradford (1976), using BSA as standard.

Reconstitution of tellurite-damaged [Fe-S] clusters. E. coli fum $C$ or acnA strains were exposed to $\mathrm{K}_{2} \mathrm{TeO}_{3}\left(0.5 \mu \mathrm{g} \mathrm{ml}^{-1}\right)$ for $15 \mathrm{~min}$, and crude extracts were prepared and incubated overnight under a $\mathrm{N}_{2}$ atmosphere in the presence or absence of $150 \mu \mathrm{M}$ $\mathrm{NH}_{4} \mathrm{Fe}\left(\mathrm{SO}_{4}\right)_{2}$ prior to fumarase or aconitase activity assessment. Control extracts were prepared from unexposed cells.

Table 1. Bacteria used in this study

\begin{tabular}{|c|c|c|}
\hline E. coli strain & Relevant genotype & Source or reference \\
\hline OD570 & BW25113 isogenic, fumC, $\mathrm{Cm}^{\mathrm{R}}$ & J. Imlay, USA \\
\hline JWK0114 & BW25113 isogenic, $a c n B, \mathrm{Km}^{\mathrm{R}}$ & Nara Institute, Japan \\
\hline PGS57 & $\begin{array}{l}\text { BW25113 isogenic, fumA fumB fumC; carries the fumA gene cloned in } \\
\text { pGS57 expression vector, Tet }{ }^{\mathrm{R}}\end{array}$ & J. Imlay, USA \\
\hline pET-fumA & E. coli BL21(DE3), transformed with pET-TOPO/101-fumA, Amp ${ }^{\mathrm{R}}$ & This work \\
\hline
\end{tabular}


Table 2. Primers used in this study

\begin{tabular}{|lll|}
\hline Primer & Orientation & \multicolumn{1}{c|}{ Sequence $\left(\mathbf{5}^{\prime} \mathbf{3}^{\prime} \mathbf{)}\right.$} \\
\hline fumCF & Direct & GTCGTTCCCCATCACCTGACAGCA \\
fumAR & Reverse & GCGTAGCAGATGAACTGGCAGTCA \\
acnAF & Direct & GTTTCCATGCTTATCCCGGATGTA \\
acnAR & Reverse & TTCGACCAACTCGACCTGATCTTC \\
csdBF & Direct & ATGTCTCCAACGTGCTTGGC \\
csdBR & Reverse & TTGGGTGTACCGGCTTCAAA \\
soxSF & Direct & TTACAGGCGGTGGCGATAATCGCT \\
soxSR & Reverse & CGAGCATATTGACCAGCCGCTTAA \\
sodAF & Direct & CCTGCCATCCCTGCCGTATGCTTA \\
sodAR & Reverse & TCGATAGCCGCTTTCAGGTCACCC \\
rrsHF & Direct & ATGACCAGCCACACTGGAAC \\
rrs $\mathrm{R}$ & Reverse & TGACTTAACAAACCGCCTGC \\
fumA- & Direct & CACCATGTCAAACAAACCCTTTCA \\
TOPO & & \\
fumA- & Reverse & TTTCACACAGCGGGTGC \\
TOPO & & \\
\hline
\end{tabular}

Detection of free $\mathbf{F e}^{\mathbf{2 +}}$ and EPR analysis. Purified FumA (15 $\left.\mu \mathrm{g}\right)$ was exposed for $45 \mathrm{~min}$ to the ITRS, and $2,2^{\prime}$-dipyridyl $(200 \mu \mathrm{M})$ was added to the mix. Formation of the 2,2'-dipyridyl- $\mathrm{Fe}^{2+}$ complex was monitored at $520 \mathrm{~nm}$ and released ferrous ion was estimated $\left(\varepsilon=5.2 \mathrm{mM}^{-1} \mathrm{~cm}^{-1}\right)$ as described by Fu et al. (1994).

EPR spectra were obtained after treating purified FumA (in $20 \mathrm{mM}$ potassium phosphate $\mathrm{pH} 7.4,10 \%$ glycerol) for defined times with the ITRS or directly with $100 \mathrm{mM} \mathrm{H}_{2} \mathrm{O}_{2}$. Mixtures were frozen in quartz EPR tubes with liquid nitrogen and analysed as described by Djaman et al. (2004). $\mathrm{H}_{2} \mathrm{O}_{2}$ exposure was ended by addition of catalase $\left(200 \mathrm{U} \mathrm{ml}^{-1}\right.$, final concentration).

\section{RESULTS}

\section{Effect of tellurite on $E$. coli fumarase and aconitase activities}

Aconitase and fumarase, two key metabolic enzyme activities, were used as models to study the effect of tellurite on E. coli [4Fe-4S] cluster-containing dehydratases. ROS-sensitive FumA and AcnB and ROS-resistant FumC and AcnA were assayed in crude cell-free extracts from tellurite-exposed cells grown in the presence or absence of oxygen.

To assess the effect of tellurite on ROS-sensitive dehydratases, total fumarase and aconitase activities were determined in cell-free extracts from E. coli fumC and $E$. coli acnA, respectively. While fumarase and aconitase activities were inhibited by $80 \%$ in cells grown aerobically, no inhibitory effect was observed under anaerobic conditions (Fig. 1a, b). To rule out that the observed telluritemediated dehydratase inhibition could be due to a general blockage of the translation machinery, a control was carried out in which FumA activity was determined in extracts of chloramphenicol-treated E. coli exposed or unexposed to potassium tellurite (see supplementary Fig. S1, available with the online version of this paper). The same level of FumA was observed irrespective of chloramphenicol treatment, suggesting that the tellurite inhibitory effect is not related to enzyme translation.

FumC and AcnA were also assayed in cell-free extracts from E. coli exposed for defined times to $\mathrm{K}_{2} \mathrm{TeO}_{3}$. Extracts from wild-type cells previously treated to disable oxidation-sensitive FumA and FumB isoforms were used to analyse FumC, while extracts from $a c n B$ strains were used to analyse AcnA activity. Both FumC and AcnA activities increased in tellurite-treated cells as compared to unexposed controls (Fig. 2a, b). An approximately fourfold induction at $15 \mathrm{~min}$ post exposure was observed for both dehydratases. In addition, approximately $10 \%$ and $30 \%$ induction of acnA and fumC transcription, respectively, was observed in tellurite-exposed cells (Table 3 ). Increased transcription of two other genes involved in the oxidative stress response, soxS and $\operatorname{sodA}$, was also observed in cells exposed to tellurite or MV (positive control). Interestingly, tellurite also strongly induced transcription of sufS (also known as $c s d B$ ), which encodes a cysteine desulfurase
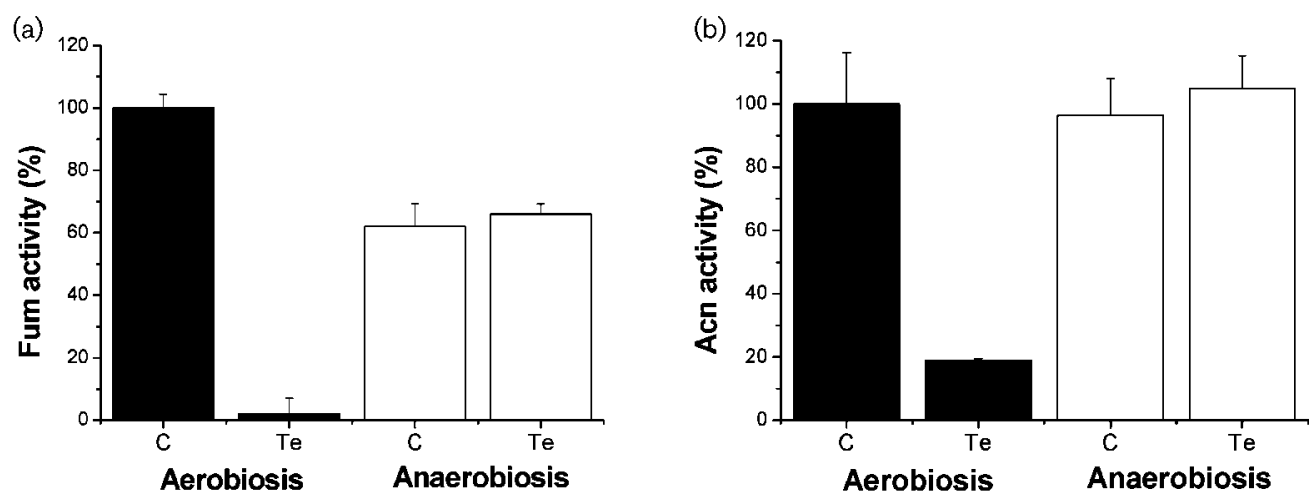

Fig. 1. Inhibition of $E$. coli ROS-sensitive dehydratases by $\mathrm{K}_{2} \mathrm{TeO}_{3}$. Total fumarase (a) and aconitase (b) activity was determined in cell-free extracts from $E$. coli cells exposed to $\mathrm{K}_{2} \mathrm{TeO}_{3}\left(0.5 \mu \mathrm{g} \mathrm{ml}^{-1}\right)$ for $30 \mathrm{~min}(\mathrm{Te})$ in aerobic (solid bars) and anaerobic conditions (open bars). C, unexposed control. Bars represent SD $(n=4)$. 

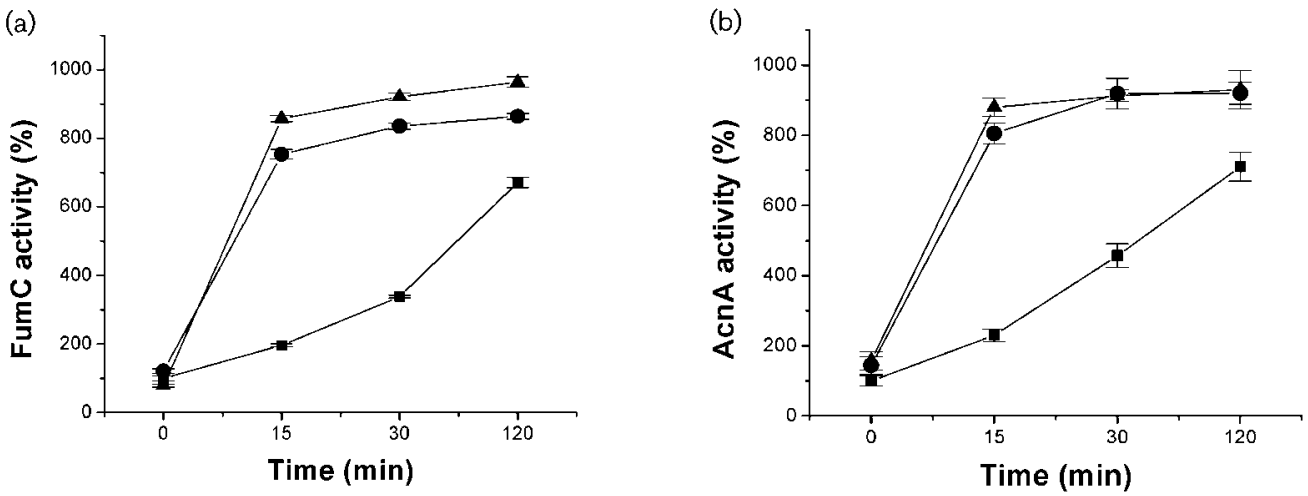

Fig. 2. FumC and $A c n A$ induction in tellurite-exposed E. coli. FumC and $A c n A$ specific activities were determined in cell-free extracts from $E$. coli wild-type and acn $B$, respectively. Cells were exposed for the indicated times to $\mathrm{K}_{2} \mathrm{TeO}_{3}\left(0.5 \mu \mathrm{g} \mathrm{ml}{ }^{-1}, \mathbf{0}\right)$ or methyl viologen $\left(100 \mu \mathrm{g} \mathrm{ml}^{-1}, \mathbf{\Delta}\right) ; \mathbf{\square}$, unexposed controls. Bars represent SD $(n=4)$.

involved in repairing oxidatively damaged [Fe-S] clusters (Zheng et al., 2001; Outten et al., 2003). In contrast, the housekeeping $r r s H$ gene (internal control), encoding $16 \mathrm{~S}$ rRNA, showed a minor transcriptional repression in exposed cells (Table 3).

\section{Reconstitution of tellurite-damaged aconitase and fumarase activities}

Fumarase and aconitase reconstitution experiments were carried out using crude extracts of $E$. coli cells lacking the oxidative-resistant isoforms FumC and AcnA, respectively. Enzyme activities that had been damaged by tellurite in vivo were slightly restored upon overnight incubation of crude extracts in the absence of oxygen but fully recovered if $\mathrm{Fe}^{2+}$ ions were present (Fig. 3a, b). Aconitase [4Fe-4S] cluster reconstitution was hardly achieved - even in the presence of $\mathrm{Fe}^{2+}$ - in crude extracts of E. coli lacking $c s d B$, a gene involved in the repair of [Fe-S] clusters (Fig. S2). Taken together, these results suggest that tellurite most probably affects these enzymes by disabling their [4Fe-4S] clusters.

Table 3. Tellurite-mediated transcriptional induction of antioxidant genes in E. coli

\begin{tabular}{|c|c|c|c|}
\hline \multirow[t]{2}{*}{ Gene } & \multicolumn{3}{|c|}{ RNA (ng) } \\
\hline & Control & $\begin{array}{c}\mathrm{K}_{2} \mathrm{TeO}_{3} \\
\left(0.5 \mu \mathrm{g} \mathrm{ml}^{-1}\right)\end{array}$ & $\begin{array}{c}\text { MV } \\
\left(100 \mu \mathrm{g} \mathrm{ml}^{-1}\right)\end{array}$ \\
\hline $\operatorname{soxS}$ & $241 \pm 11$ & $438 \pm 14$ & $472 \pm 14$ \\
\hline $\operatorname{sod} A$ & $338 \pm 7$ & $399 \pm 12$ & $410 \pm 15$ \\
\hline fumC & $227 \pm 9$ & $290 \pm 9$ & $335 \pm 7$ \\
\hline $\operatorname{acn} A$ & $310 \pm 8$ & $343 \pm 6$ & $370 \pm 5$ \\
\hline$c s d B$ & $90 \pm 16$ & $193 \pm 12$ & $207 \pm 10$ \\
\hline$r r s H$ & $338 \pm 8$ & $315 \pm 8$ & $309 \pm 6$ \\
\hline
\end{tabular}

\section{In vitro tellurite reduction results in FumA inhibition}

Purified E. coli FumA lost almost $90 \%$ of activity when exposed to the ITRS (Fig. 4a). However, no effect was observed if the enzyme was treated with tellurite alone (not shown), a result suggesting that superoxide radical being produced during incubation of FumA with ITRS could be involved in the tellurite-mediated damage to the enzyme. To assess if superoxide is actually involved in FumA inhibition, a control was carried out in which purified FumA was incubated for 30 min with a known superoxidegenerating system (xanthine/xanthine oxidase). Fig. 4(a) shows that FumA exposed to either superoxide-generating system lost $\sim 90 \%$ activity under aerobic conditions as compared to untreated controls. To obtain further evidence that superoxide is involved in FumA damage, the same experiment was carried under anaerobic conditions. No significant differences in enzyme activity were observed in superoxide-exposed and control FumA in the absence of oxygen (Fig. 4b). Enzyme activity of the ITRSdamaged purified FumA was almost fully recovered after incubating in the presence of DTT and ferrous ion under anaerobic conditions (Fig. 4c), indicating that this in vitro FumA inhibition involves the disabling of its [4Fe-4S] cluster.

To confirm this last assumption ferrous ion released from purified FumA after exposure to the ITRS was assessed using the highly specific $\mathrm{Fe}^{2+}$ chelator 2,2'-bipyridyl. The enzyme lost over $90 \%$ of its $\mathrm{Fe}^{2+}$ upon ITRS exposure as compared to unexposed controls (not shown), confirming that tellurite-mediated enzyme inactivation involves [4Fe$4 S$ ] cluster disruption. Further support for these findings came from EPR experiments in which purified FumA was incubated for 10 and $30 \mathrm{~min}$ with the ITRS or for 5 and 30 min with $\mathrm{H}_{2} \mathrm{O}_{2}$ (control). At short incubation times the characteristic signal corresponding to the paramagnetic, inactive $[3 \mathrm{Fe}-4 \mathrm{~S}]^{+}$cluster was observed (Fig. S3), 
(a)

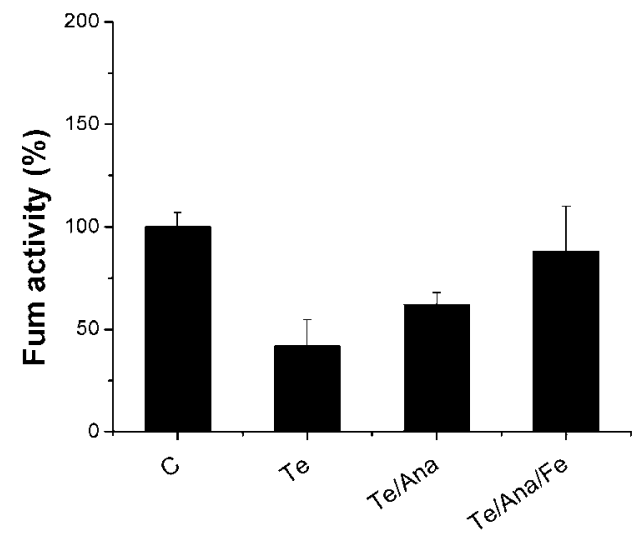

(b)

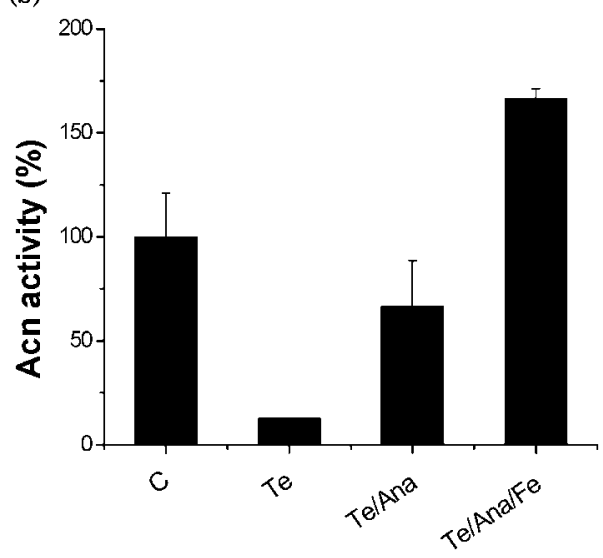

Fig. 3. Reconstitution of tellurite-damaged fumarase (a) and aconitase (b) activities in crude extracts of $E$. coli cells exposed for $15 \mathrm{~min}$ to $\mathrm{K}_{2} \mathrm{TeO}_{3}\left(0.5 \mu \mathrm{g} \mathrm{ml}^{-1}\right)$. C, unexposed control; Te, tellurite-exposed; Te/Ana, tellurite-exposed, incubated in anaerobic conditions for $12 \mathrm{~h}$; Te/Ana/Fe, as Te/Ana, but in the presence of $\mathrm{Fe}^{+2}$ ions. Bars represent SD $(n=3)$.

strengthening the idea of an oxidatively mediated loss of $\mathrm{Fe}^{2+}$ from the enzyme.

\section{DISCUSSION}

The ultimate molecular mechanism underlying bacterial tellurite toxicity is not fully understood. Preliminary evidence was obtained years ago from studies aimed at identifying and characterizing genetic bacterial tellurite resistance $\left(T e l^{r}\right)$ determinants. For example, the cysK gene of Geobacillus stearothermophilus V, encoding cysteine synthase, functions as a $\mathrm{Tel}^{\mathrm{r}}$ determinant in E. coli (Vásquez et al., 2001). Other groups have also reported that expression of cysteine synthase genes from E. coli (Alonso et al., 2000), Rhodobacter sphaeroides (O'Gara et al., 1997), Staphylococcus aureus (Lithgow et al., 2004) and Azospirillum brasilense (Ramírez et al., 2006) conferred resistance to potassium tellurite. It was shown that the iscS gene from $G$. stearothermophilus $\mathrm{V}$, encoding a cysteine desulfurase, also conferred tellurite resistance in E. coli (Tantaleán et al., 2003). In addition, E. coli lacking $c s d A$, $c s d B$ or iscS was shown to be hypersensitive to $\mathrm{K}_{2} \mathrm{TeO}_{3}$ (Rojas \& Vásquez, 2005). Cysteine synthase (CysK) and cysteine desulfurase (IscS) enzymes are probably functionally related since IscS catalyses the desulfurization of Lcysteine and participates in the incorporation of the released sulfur atom into ROS-damaged [Fe-S] clusters (Zheng et al., 1993, 1994). Cysteine desulfurases from the iscAUS and sufABCDSE operons of $E$. coli are involved in a complex machinery responsible for de novo synthesis as well as for the repair of ROS-damaged [Fe-S] clusters (Djaman et al., 2004; Loiseau et al., 2003). Thus, it was tempting to speculate that repair of [Fe-S] clusters may represent a bacterial defensive mechanism against ROS elicitors such as potassium tellurite. (a)

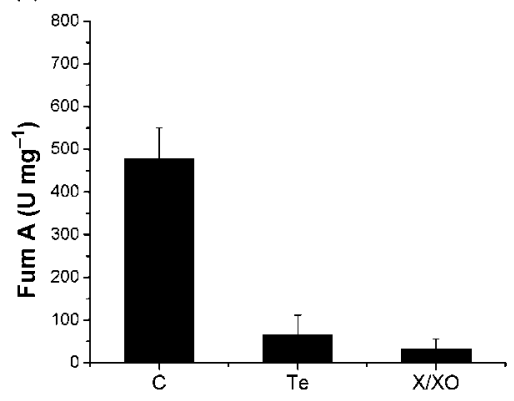

(b)

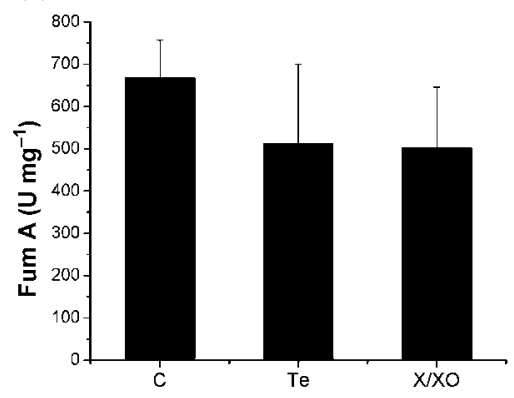

(c)

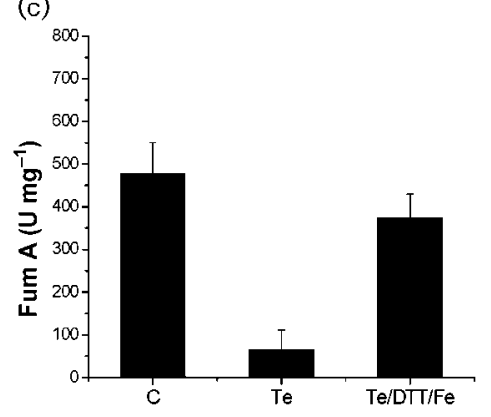

Fig. 4. Inhibition of FumA by an ITRS. Purified FumA was incubated for $30 \mathrm{~min}$ in aerobic conditions with ITRS (Te) or xanthine/ xanthine oxidase $(X / X O)$ in aerobic (a) and anaerobic conditions (b). (c) Reconstitution of tellurite-damaged purified FumA: FumA exposed to ITRS (Te); reconstitution in anaerobic conditions in the presence of dithiothreitol (DTT) and Fe $\mathrm{F}^{2+}$ ions (Te/DTT/Fe). C represents unexposed FumA in all panels. 
Previous results from our laboratory showed that telluriteexposed E. coli exhibited decreased fumarase and aconitase activities and that in vivo and in vitro reduction of tellurite resulted in superoxide production (Calderón et al., 2006; Pérez et al., 2007). Since some of the main intracellular superoxide targets are [4Fe-4S] cluster-containing enzymes (Imlay, 2003), we speculated that superoxide generated as a consequence of tellurite reduction could be involved in inhibiting aconitase and fumarase. In this work the oxidizing ability of tellurite on $A c n B$ and FumA was evaluated using acnA and fumC cells, respectively. The observed inhibition of AcnB and FumA was almost entirely dependent on the presence of oxygen, suggesting that tellurite's inhibitory effect was mediated by ROS (Fig. 1). Subsequent in vitro experiments demonstrated that tellurite-mediated FumA inactivation involves $\mathrm{Fe}^{2+}$ release from the enzyme, a common feature observed in ROS-damaged [4Fe-4S] clusters (Flint et al., 1993a, b; Imlay, 2003).

To further demonstrate that tellurite reduction drives FumA inactivation, the enzyme was exposed to an ITRS (Calderón et al., 2006; Pérez et al., 2007). As a positive control, FumA was independently exposed to xanthine/ xanthine oxidase, a well known superoxide-generating system. In both cases FumA activity was reduced over $90 \%$ in the presence of oxygen, a result that was not observed under anaerobic conditions (Fig. 4a, b). The direct involvement of superoxide in FumA inactivation was demonstrated using SOD. Fig. S4 clearly shows that FumA is protected from ITRS inactivation in the presence of superoxide dismutase.

Increased intracellular superoxide levels result in augmented SodA activity, a consequence of SoxRS-mediated $\operatorname{sodA}$ transcriptional activation (Imlay, 2003). Although $\operatorname{sodA}$ induction was previously observed in tellurite-exposed $E$. coli (Pérez et al., 2007) here we show that it occurs at the transcriptional level. Transcription of acnA, fumC and sufS was also found to be increased in tellurite-exposed cells (Table 3).

Brown et al. (2002) demonstrated that inactive forms of $[4 \mathrm{Fe}-4 \mathrm{~S}]^{2+}$ clusters, $[3 \mathrm{Fe}-4 \mathrm{~S}]^{+}$, are paramagnetic and can be detected in vivo by paramagnetic resonance spectroscopy (EPR). In this work, EPR was used to demonstrate the presence of the inactive $[3 \mathrm{Fe}-4 \mathrm{~S}]^{+}$form in purified FumA exposed to ITRS (Fig. S3). FumA exposure to tellurite (or $\mathrm{H}_{2} \mathrm{O}_{2}$, positive control) for 30 min caused the characteristic signal at 3200 gauss $(0.32 \mathrm{~T})$ to disappear, which is most probably due to overexposure of the ROSsensitive cluster generating non-paramagnetic structures (Djaman et al., 2004).

Finally, it is worth making some comments about the high toxicity of $\mathrm{K}_{2} \mathrm{TeO}_{3}$ for micro-organisms. As was observed by Turner et al. $(1999,2001)$ tellurite causes thiol oxidation, compromising seriously the redox balance of the cell. Moreover, superoxide generated as byproduct of tellurite reduction (Pérez et al., 2007) may result in increased intracellular $\mathrm{H}_{2} \mathrm{O}_{2}$ levels, either by specific superoxide dismutation by SOD or by accidental transfer of electrons to $\mathrm{O}_{2}^{-}$during auto-oxidation of respiratory dehydrogenases (Massey, 1994; Messner \& Imlay, 1999, 2002). Since $\mathrm{H}_{2} \mathrm{O}_{2}$ also oxidizes [Fe-S] clusters (Storz \& Imlay, 1999), released $\mathrm{Fe}^{2+}$ can react with $\mathrm{H}_{2} \mathrm{O}_{2}$ (Fenton reaction) to generate hydroxyl radical, of which nucleic acids and membrane lipids (Refsgaard et al., 2000) are important cellular targets. In this context, increased TBARs have been found in E. coli exposed to potassium tellurite (Pérez et al., 2008). Since oxidative stress results in widespread damage of the cell, an immediate consequence is a decrease in cell viability. Several tellurite resistance determinants identified so far seem to deal with adaptive responses of a cell facing oxidative conditions. Experiments to shed further light on these interesting findings are under way in our laboratory.

\section{ACKNOWLEDGEMENTS}

The authors are indebted to Dr Thomas G. Chasteen, Sam Houston State University, Texas, USA, for critical reading of the manuscript. I. L.C., M.E.C. and F.A.A. received doctoral fellowships from Conicyt, Chile. J. M. P, A. O.E. and G. A.P. received doctoral fellowships from MECESUP, Chile. J.M.P. was also supported by Conicyt and Dicyt-USACH, Chile. This work received financial support from Grant no. 1060022 from Fondecyt and Dicyt-USACH to C.C.V.

\section{REFERENCES}

Alonso, G., Gomes, C., González, C. \& Rodríguez-Lemoine, V. (2000). On the mechanism of resistance to channel-forming colicins (PacB) and tellurite, encoded by plasmid Mip233 (IncHI3). FEMS Microbiol Lett 192, 257-261.

Borsetti, F., Tremaroli, V., Michelacci, F., Borghese, R., Winterstein, C., Daldal, F. \& Zannoni, D. (2005). Tellurite effects on Rhodobacter capsulatus cell viability and superoxide dismutase activity under oxidative stress conditions. Res Microbiol 156, 807-813.

Bradford, M. M. (1976). A rapid and sensitive method for the quantitation of microgram quantities of protein utilizing the principle of protein-dye binding. Anal Biochem 72, 248-254.

Brown, N. M., Kennedy, M. C., Antholine, W. E., Eisenstein, R. S. \& Walden, W. E. (2002). Detection of a [3Fe-4S] cluster intermediate of cytosolic aconitase in yeast expressing iron regulatory protein 1. Insights into the mechanism of Fe-S cluster cycling. J Biol Chem 277, 7246-7254.

Calderón, I. L., Arenas, F. A., Pérez, J. M., Fuentes, D. E., Araya, M. A., Saavedra, C. P., Tantaleán, J. C., Pichuantes, S. E., Youderian, P. A. \& Vásquez, C. C. (2006). Catalases are $\mathrm{NAD}(\mathrm{P}) \mathrm{H}$-dependent tellurite reductases. PLoS One 1, e70.

Djaman, O., Outten, W. \& Imlay, J. A. (2004). Repair of oxidized ironsulfur clusters in Escherichia coli. J Biol Chem 279, 44590-44599.

Flint, D. H., Smyk-Randall, E., Tuminello, J. F., Draczynska-Lusiak, B. \& Brown, O. R. (1993a). The inactivation of dihydroxy-acid dehydratase in Escherichia coli treated with hyperbaric oxygen occurs because of the destruction of its Fe-S cluster, but the enzyme remains in the cell in a form that can be reactivated. J Biol Chem 268, 2554725552.

Flint, D. H., Tuminello, J. F. \& Emptage, M. (1993b). The inactivation of $\mathrm{Fe}-\mathrm{S}$ cluster containing hydro-lyases by superoxide. J Biol Chem 268, 22369-22376. 
Fu, W., Jack, R., Morgan, T., Dean, D. \& Johnson, M. (1994). nifU gene product from Azotobacter vinelandii is a homodimer that contains two identical [2Fe-2S] clusters. Biochemistry 33, 13455-13463.

Gardner, P. R. \& Fridovich, I. (1991). Superoxide sensitivity of the Escherichia coli aconitase. J Biol Chem 266, 19328-19333.

Imlay, J. A. (2003). Pathways of oxidative damage. Annu Rev Microbiol 57, 395-418.

Kuo, C. F., Mashino, T. \& Fridovich, I. (1987). $\alpha, \beta$-Dihydroxyisovalerate dehydratase. A superoxide-sensitive enzyme. J Biol Chem 262, $4724-4727$.

Liochev, S. I. \& Fridovich, I. (1993). Modulation of the fumarases of Escherichia coli in response to oxidative stress. Arch Biochem Biophys 301, 379-384.

Lithgow, J. K., Hayhurst, E. J., Cohen, G., Aharonowitz, Y. \& Foster, S. J. (2004). Role of a cysteine synthase in Staphylococcus aureus. J Bacteriol 186, 1579-1590.

Loiseau, L., Ollagnier-de-Choudens, S., Nachin, L., Fontecave, M. \& Barras, F. (2003). Biogenesis of $\mathrm{Fe}-\mathrm{S}$ cluster by the bacterial Suf system: SufS and SufE form a new type of cysteine desulfurase. J Biol Chem 278, 38352-38359.

Massey, V. (1994). Activation of molecular oxygen by flavins and flavoproteins. J Biol Chem 269, 22459-22462.

Messner, K. R. \& Imlay, J. A. (1999). The identification of primary sites of superoxide and hydrogen peroxide formation in the aerobic respiratory chain and sulfite reductase complex of Escherichia coli. $J$ Biol Chem 274, 10119-10128.

Messner, K. R. \& Imlay, J. A. (2002). Mechanism of superoxide and hydrogen peroxide formation by fumarate reductase, succinate dehydrogenase, and aspartate oxidase. J Biol Chem 277, 42563-42571.

O'Gara, J. P., Gomelsky, M. \& Kaplan, S. (1997). Identification and molecular genetic analysis of multiple loci contributing to high-level tellurite resistance in Rhodobacter sphaeroides 2.4.1. Appl Environ Microbiol 63, 4713-4720.

Outten, F. W., Wood, M. J., Muñoz, F. M. \& Storz, G. (2003). The SufE protein and the SufBCD complex enhance SufS cysteine desulfurase activity as part of a sulfur transfer pathway for $\mathrm{Fe}-\mathrm{S}$ cluster assembly in Escherichia coli. J Biol Chem 278, 45713-45719.

Pérez, J. M., Calderón, I. L., Arenas, F. A., Fuentes, D. E., Pradenas, G. A., Fuentes, E. L., Sandoval, J. M., Castro, M. E., Elías, A. O. \& Vásquez, C. C. (2007). Bacterial toxicity of potassium tellurite: unveiling an ancient enigma. PLoS One 2, e211.

Pérez, J. M., Arenas, F. A., Pradenas, G. A., Sandoval, J. M. \& Vásquez, C. C. (2008). Escherichia coli YqhD exhibits aldehyde reductase activity and protects from the harmful effect of lipid peroxidation-derived aldehydes. J Biol Chem 283, 7346-7353.

Ramírez, A., Castañeda, M., Xiqui, M. L., Sosa, A. \& Baca, B. E. (2006). Identification, cloning and characterization of $c y s K$, the gene encoding O-acetylserine (thiol)-lyase from Azospirillum brasilense, which is involved in tellurite resistance. FEMS Microbiol Lett 261, 272-279.

Refsgaard, H. H., Tsai, L. \& Stadtman, E. R. (2000). Modifications of proteins by polyunsaturated fatty acid peroxidation products. Proc Natl Acad Sci U S A 97, 611-616.

Rojas, D. M. \& Vásquez, C. C. (2005). Sensitivity to potassium tellurite of Escherichia coli cells deficient in CSD, CsdB and IscS cysteine desulfurases. Res Microbiol 156, 465-471.

Sambrook, J., Fritsch, E. F. \& Maniatis, T. (1989). Molecular Cloning: a Laboratory Manual, 2nd edn. Cold Spring Harbor, NY: Cold Spring Harbor Laboratory.

Storz, G. \& Imlay, J. A. (1999). Oxidative stress. Curr Opin Microbiol 2, 188-194.

Tantaleán, J. C., Araya, M. A., Pichuantes, S. E., Saavedra, C. P., Fuentes, D. E., Pérez, J. M., Calderón, I. L. \& Vásquez, C. C. (2003). The Geobacillus stearothermophilus $\mathrm{V}$ isc $\mathrm{S}$ gene, encoding cysteine desulfurase, confers resistance to potassium tellurite in Escherichia coli K-12. J Bacteriol 185, 5831-5837.

Taylor, D. E. (1999). Bacterial tellurite resistance. Trends Microbiol 7, 111-115.

Tremaroli, V., Fedi, F. \& Zannoni, D. (2006). Evidence for a telluritedependent generation of reactive oxygen species and absence of a tellurite-mediated adaptive response to oxidative stress in cells of Pseudomonas pseudoalcaligenes KF707. Arch Microbiol 187, 127-135.

Turner, R. J., Weiner, J. \& Taylor, D. E. (1999). Tellurite-mediated thiol oxidation in Escherichia coli. Microbiology 145, 2549-2557.

Turner, R. J., Aharonowitz, Y., Weiner, J. \& Taylor, D. E. (2001). Glutathione is a target in tellurite toxicity and is protected by tellurite resistance determinants in Escherichia coli. Can J Microbiol 47, 33-40.

Varghese, S., Tang, Y. \& Imlay, J. A. (2003). Contrasting sensitivities of Escherichia coli aconitases A and B to oxidation and iron depletion. J Bacteriol 185, 221-230.

Vásquez, C. C., Saavedra, C. P., Loyola, C. A., Araya, M. A. \& Pichuantes, S. E. (2001). The product of the cysK gene of Bacillus stearothermophilus $\mathrm{V}$ mediates potassium tellurite resistance in Escherichia coli. Curr Microbiol 43, 418-423.

Zheng, L., White, R., Cash, V., Jack, R. \& Dean, D. (1993). Cysteine desulfurase activity indicates a role for NifS in metallocluster biosynthesis. Proc Natl Acad Sci U S A 90, 2754-2758.

Zheng, L., White, R., Cash, V. \& Dean, D. (1994). Mechanism for the sulfurization of L-cysteine catalyzed by the nifS gene product. Biochemistry 33, 4714-4720.

Zheng, M., Wang, X., Templeton, L., Smulski, D., LaRossa, R. \& Storz, G. (2001). DNA microarray-mediated transcriptional profiling of the Escherichia coli response to hydrogen peroxide. J Bacteriol 183, $4562-4570$.

Edited by: J. Moir 